\title{
Working memory capacity compensates hearing related phonological processing deficit
}

\author{
Elisabet Classon, Mary Rudner and Jerker Rönnberg
}

\section{Linköping University Post Print}

N.B.: When citing this work, cite the original article.

Original Publication:

Elisabet Classon, Mary Rudner and Jerker Rönnberg, Working memory capacity compensates hearing related phonological processing deficit, 2013, Journal of Communication Disorders, (46), 1, 17-29.

http://dx.doi.org/10.1016/j.jcomdis.2012.10.001

Copyright: Elsevier

http://www.elsevier.com/

Postprint available at: Linköping University Electronic Press

http://urn.kb.se/resolve?urn=urn:nbn:se:liu:diva-74276 
Running head: WORKING MEMORY COMPENSATES

\title{
Working memory compensates for hearing related phonological
}

$$
\text { processing deficit* }
$$

\author{
Elisabet Classon ${ }^{\mathrm{a}, \dagger}$, Mary Rudner ${ }^{\mathrm{a}, 1}$, Jerker Rönnberg ${ }^{\mathrm{a}, 2}$
}

${ }^{a}$ Linnaeus Centre HEAD, The Swedish Institute for Disability Research, Department of Behavioural Sciences and Learning, Linköping University, Linköping, Sweden.

\footnotetext{
Abbreviations: ELU, ease of language understanding model; WMC, working memory capacity; R+, rhyming; R-, non-rhyming; O+, orthographically similar; O-, orthographically dissimilar; RAMBPHO, rapid automatic binding of phonology; NAM, neighbourhood activation model; SOA, stimulus onset asynchrony; ERP, event-related potentials; $\mathrm{HI}$, hearing impairment; BestEarPTA, best ear pure tone average; $\mathrm{NH}$, normal hearing; RSPM, Ravens standard progressive matrices; DLS, diagnostic tests of reading and writing; NH-High, normal hearing and high WMC; $\mathrm{NH}-$ Low, normal hearing and low WMC; HI-High, hearing impairment and high WMC; HI-Low, hearing impairment and low WMC.

* Corresponding author at: Department of Behavioural Sciences and Learning, Linköping University, SE-581 83 Linköping, Sweden. Tel.: +46 13 282249; fax: +46 13282145.

E-mail address: elisabet.classon@liu.se.

${ }^{1}$ Tel.: +46 13 282157; e-mail address: mary.rudner@liu.se.

${ }^{2}$ Tel.: +46 13 282107; e-mail address: jerker.ronnberg@liu.se.
} 


\title{
WORKING MEMORY COMPENSATES
}

\begin{abstract}
Acquired hearing impairment is associated with gradually declining phonological representations. According to the Ease of Language Understanding (ELU) model, poorly defined representations lead to mismatch in phonologically challenging tasks. To resolve the mismatch, reliance on working memory capacity (WMC) increases. This study investigated whether WMC modulated performance in a phonological task in individuals with hearing impairment. A visual rhyme judgment task with congruous or incongruous orthography, followed by an incidental episodic recognition memory task, was used. In participants with hearing impairment, WMC modulated both rhyme judgment performance and recognition memory in the orthographically similar non-rhyming condition; those with high WMC performed exceptionally well in the judgment task, but later recognized few of the words. For participants with hearing impairment and low WMC the pattern was reversed; they performed poorly in the judgment task but later recognized a surprisingly large proportion of the words. Results indicate that good WMC can compensate for the negative impact of auditory deprivation on phonological processing abilities by allowing for efficient use of phonological processing skills. They also suggest that individuals with hearing impairment and low WMC may use a non-phonological approach to written words, which can have the beneficial side effect of improving memory encoding.
\end{abstract}

Keywords: hearing impairment; phonology; working memory capacity; episodic recognition 
WORKING MEMORY COMPENSATES

\section{Introduction}

Individuals with acquired hearing impairment tend to fall behind normal hearers in phonologically challenging tasks like visual rhyme judgment. This has been attributed to gradually declining phonological representations. As the years with impoverished auditory input increase, mental representations of both speech sounds and non-speech sounds become less well-defined (Andersson, 2002; Andersson \& Lyxell, 1998; Lazard, Giraud, Truy \& Lee, 2011; Lazard, Lee, Gaebler, Kell, Truy \& Giraud, 2010; Lee, Truy, Mamou, Sappey-Marinier \& Giraud, 2007; Lyxell et al., 1998; Lyxell, Andersson, Borg \& Ohlsson, 2003; Lyxell, Rönnberg \& Samuelsson, 1993). According to the Ease of Language Understanding model (ELU, Rönnberg, 2003; Rönnberg, Rudner, Foo \& Lunner, 2008) poorly defined speech sound representations will lead to mismatch in phonologically challenging tasks. Explicit resources, such as working memory capacity (WMC), are seen as crucial in resolving mismatch. In this study we explored whether individual WMC modulated visual rhyme judgment performance in individuals with acquired hearing impairment. In addition, as increased occurrence of mismatch might interfere with episodic memory encoding, we used an incidental episodic recognition memory task to investigate whether hearing related mismatch affected episodic memory encoding of words from the rhyme judgment task.

\subsection{Rhyme judgment and working memory}

Working memory is a limited resource employed in simultaneous processing and storage of information (Baddeley, 2000, Daneman \& Carpenter, 1980; Daneman \& Merikle, 1996). The dual processing function separates working memory from short-term memory, semantic long- 
WORKING MEMORY COMPENSATES

term memory and episodic memory that are typically more focused on sole storage of information over different time periods or, in the case of episodic memory, storage of events tagged for a particular time and place (Tulving, 2002). Working memory is instead engaged in the online process of holding information while manipulating it during cognitively demanding tasks such as visual rhyme judgments.

Deciding whether pairs of written words rhyme or not is complex and involves spelling-to-sound re-coding, monitoring, phonological extraction of the rime portion and active maintenance in working memory while assessing phonological similarity. Rhyme task wordpairs can rhyme $(\mathrm{R}+)$ or not $(\mathrm{R}-)$ and be orthographically similar $(\mathrm{O}+)$ or dissimilar $(\mathrm{O}-)$. In this paper, rhymes are defined as words whose final stressed vowel and following sounds are all identical. Rhyme judgments are most difficult when the phonological and orthographic cues give incongruous information, i.e. orthographically dissimilar rhymes (R+O-, e.g. moose - juice) and orthographically similar non-rhymes (R-O+, e.g. bead - dead) (Johnston \& McDermott, 1986; Kramer \& Donchin, 1987; Polich, McCarthy, Wang \& Donchin, 1983; Rugg \& Barrett, 1987). In these conditions there is a need to rely heavily on phonological representations stored in longterm memory and to perform operations on these representations to solve the task.

This is true whether the task is in English or, as in the present study, in Swedish. Swedish is however more consistent in its grapheme-to-phoneme correspondence than English (Seymour, Aro \& Erskine, 2003). A specific letter combination can typically only be pronounced in one way. Consequently, there are few direct Swedish equivalents of English word-pairs such as beaddead where the rime is orthographically identical but phonologically dissimilar. Instead, Swedish R-O+ word-pairs used in rhyme judgment tasks have orthographically similar, but not identical, endings, e.g. sant - saft, ([san:t] - [saf:t]) (Andersson, 2002; Andersson \& Lyxell, 1998; 
WORKING MEMORY COMPENSATES

Andersson, Lyxell, Rönnberg \& Spens, 2001; Lyxell et al., 1998; Lyxell et al., 1993). They are both orthographically and phonologically similar, but non-rhyming. This fine-grained phonological difference makes them difficult to separate phonologically and puts high demands on the ability to make phonological comparisons and analyse representations in working memory.

More specifically, in rhyme judgment of serially presented words, the first word will be the basis for expectancies about the second word, generated before the second word is actually presented (Baum \& Leonard, 1999; McQueen \& Serrano, 2005). The predicted words will predominantly be rhymes. In $\mathrm{R}-\mathrm{O}+$ the prediction will not match the second word as it does not rhyme with the first word. The predictions have to be abandoned and rapid access made to the phonology of the second word while simultaneously maintaining a representation of the first word (Johnstone \& McDermott, 1986). Due to the phonological and orthographic similarity of the two representations further analyses and cross-checking might need to be carried out before a decision can be made.

Further, the Swedish words used in R-O+ word-pairs may have irregular or regular spelling. Where spelling is irregular, stored phonological representations at the syllable or whole word level need to be relied on; where it is regular, their phonology can be also be accessed via letter-to-sound conversion. The R-O+ condition is therefore considered to be primarily a measure of the ability to perform operations on phonological representations (Andersson, 2002).

The demand on WMC is likely to be even larger when the time interval between presentations of the words is long than when it is short. A long time interval allows for generation of expectancies (Baum \& Leonard, 1999; McQueen \& Serrano, 2005; Neely, 1977; 
WORKING MEMORY COMPENSATES

Neely, Keefe \& Ross, 1989) and at the same time the representation of the first word will have time to fade.

Although Swedish is more consistent than English in its grapheme-to-phoneme mapping, it is not consistent in phoneme-to-grapheme mapping. Thus, rhyming words may be spelt differently and it is possible to construct Swedish R+O- word-pairs (e.g. helg - välj, [hel:j] - [vel:j]) that are equivalent to such pairs in English (e.g. moose - juice). In contrast to $\mathrm{R}-\mathrm{O}+$ word-pairs, $\mathrm{R}+\mathrm{O}$ - word-pairs tap the precision of phonological representations more directly. Because of the irregular spelling of these words their pronunciation is not accessible via spelling-to-sound conversion. Rather, judgments need to be based on phonological representations already stored in long-term memory (Andersson, 2002). Words in this condition rhyme which means that predictions generated by the first word will more likely match the second word and reduce the need for further analyses and working memory engagement (Hillinger, 1980; Johnstone \& McDermott, 1986).

It is in these two incongruous rhyme conditions that individuals with hearing impairment are at a disadvantage as compared to individuals with normal hearing (Andersson, 2002; Andersson \& Lyxell, 1998; Lyxell et al., 1998; Lyxell et al., 1993). Compromised phonological representations interfere with rhyme judgment in both conditions, but it is likely that the possibility to compensate by recruiting explicit resources is larger in $\mathrm{R}-\mathrm{O}+$ than in $\mathrm{R}+\mathrm{O}-$.

\section{2. $E L U$}

Rönnberg, (2003) and Rönnberg, et al. (2008) proposed a model for Ease of Language Understanding, the ELU model. It aims to describe the cognitive mechanisms behind language 
WORKING MEMORY COMPENSATES

understanding in challenging conditions, i.e. when perception is compromised due to hearing impairment or suboptimal signal processing in a hearing instrument, or when the linguistic signal in itself is degraded. The ELU model proposes a reciprocal relationship between implicit bottomup and explicit top-down processes that is modulated by a general capacity working-memory system for processing and storage of information.

More specifically, the ELU model assumes that processing of perceived linguistic information involves an episodic buffer for the Rapid, Automatic and Multimodal Binding of PHOnological information (RAMBPHO). The extracted phonological input in the language signal is matched to stored phonological representations in semantic long-term memory. Under favorable conditions, the matching process is fast and implicit, resulting in effortless lexical access or word decision. However, when conditions are less favorable, e.g. when listening to speech in noise, or when phonological representations are poorly specified, the possibility of mismatch between input and stored representations increases. When mismatch occurs, the model proposes that explicit resources, such as the processing and storage functions of working memory, are recruited to resolve the ambiguity.

The model is connected to capacity theory of working memory (Daneman \& Carpenter, 1980) but adds an emphasis on phonological processing and long-term memory interactions, and assumes specific roles for implicit and explicit processing mechanisms. RAMBPHO focuses on the integration of phonological information from different sources and thus has certain similarities with the episodic buffer introduced by Baddeley (2000) into one of the major component models of working memory (Baddeley, 2000). However, unlike Baddeleys model, the ELU is geared towards the communicative outcome, i.e. language understanding, rather than working memory as such (Rudner \& Rönnberg, 2008). It differs from models of speech 
WORKING MEMORY COMPENSATES

perception (e.g., the TRACE model, McClelland \& Elman, 1986; the Cohort model, MarslenWilson, 1987; and the NAM model, Luce \& Pisoni, 1998) in its assumption that explicit WMC is called for when there is mismatch between language input and cognitive representations. The fact that the need for explicit resources is restricted to mismatch situations represents a processing economy aspect of the ELU model.

In an actual dialogical situation, the demand on working memory resources will vary from time to time but, generally speaking, the model predicts that individual WMC will modulate performance under mismatch conditions (Stenfelt \& Rönnberg, 2009). The prediction has been supported in research on auditory speech understanding in noise, e.g. when the input is degraded or distorted by advanced signal processing in hearing aids. High cognitive capacity is associated with better speech recognition under challenging listening conditions in individuals with hearing impairment (Gatehouse, Naylor \& Elberling, 2006; Lunner, 2003; Lunner \& Sundewall-Thorén, 2007; Rudner, Foo, Rönnberg \& Lunner, 2007; Rudner, Rönnberg \& Lunner, 2011) and better memory processing of heard information ( $\mathrm{Ng}$, Rudner, Lunner, Pedersen \& Rönnberg, submitted; Rönnberg, Rudner, Lunner \& Stenfelt, submitted). Under less taxing conditions, cognitive capacity no longer correlates with speech understanding (Lunner \& Sundewall-Thorén, 2007).

However, the proposal that explicit processes can compensate for mismatch due to degraded phonological representations can also be directly investigated by analyzing the role of WMC in visual rhyme judgment performance (i.e. optimal input) of participants with or without severe acquired hearing impairment (i.e. with degraded or normal phonological representations).

The first research question addressed in the present study is whether individual WMC modulates visual rhyme judgment performance in participants with hearing impairment. 
WORKING MEMORY COMPENSATES

Participants with a severe acquired hearing impairment for at least 10 years and a matched normal hearing control group were subdivided into high and low WMC subgroups by a median split of scores on a complex verbal span task. They performed a visually presented rhyme judgment task in four conditions: two congruous ( $\mathrm{R}+\mathrm{O}+$ and $\mathrm{R}-\mathrm{O}-)$ and two incongruous $(\mathrm{R}+\mathrm{O}-$ and $\mathrm{R}-\mathrm{O}+)$. The ELU model is supported if (1) WMC impacts performance more in participants with hearing impairment, i.e. where mismatch is hypothesized to occur more frequently and (2) in this group, high WMC is associated with better performance, i.e. WMC helps overcome mismatch, and (3) the effect of WMC in this group is more pronounced in the $\mathrm{R}-\mathrm{O}+$ than the $\mathrm{R}+\mathrm{O}-$ condition, i.e. in the condition where there is a relatively higher need to perform phonological operations and analyses. The stimulus onset asynchrony (SOA, the time between onsets of two consecutive stimuli) was manipulated to be either short or long to investigate whether any effect of WMC interacted with SOA. Further, due to the higher accessibility of word phonology by spelling-to-sound conversion in the R-O+ as compared to the $\mathrm{R}+\mathrm{O}-$ condition, a test of non-word reading was included. This allowed for examination of whether phonological decoding skills supported performance and in that case, whether there was an independent contribution by WMC.

\subsection{Episodic memory and hearing impairment}

Our second research question concerned episodic memory encoding. Several studies have found a relationship between hearing loss and reduced recall in visually presented episodic memory tasks (Baltes \& Lindenberger, 1997; Pearman, Friedman, Brooks \& Yesavage, 2000; Rönnberg et al., 2011; Valentijn et al., 2005). Rönnberg et al (2011) found that the degree of 
WORKING MEMORY COMPENSATES

hearing loss negatively affected episodic long-term memory despite the fact that the sample studied wore hearing aids and that chronological age was statistically accounted for. The results were interpreted in terms of the ELU model: with a larger number of mismatches occurring in everyday life communication, subsequent encoding and retrieval from episodic long-term memory occur with less frequency and with reduced memory practice as a result. Short-term memory and working memory for visually presented stimuli are however not affected by acquired hearing loss (Lyxell et al, 2003; Rönnberg et al, 2011).

It has thus been suggested that high occurrence of mismatch might impact episodic memory encoding. For example, a study investigating the effect of hearing loss on memory for speech has shown that presence of mild-to-moderate hearing loss is sufficient to reduce recall of spoken words even when cognitive load is low, and even when the words are correctly perceived (McCoy et al., 2005). Thus, the extra processing effort required by a listener with hearing impairment to perceive spoken words (i.e. overcome mismatch) occupies working memory resources otherwise available for encoding of information, which is evidenced by reduced episodic memory retrieval of the processed information (McCoy et al., 2005; Piquado, Cousins, Wingfield \& Miller, 2010; Rudner et al., 2011; Surprenant, 2007; Tun, McCoy \& Wingfield, 2009).

To our knowledge, it has not been investigated whether hearing related mismatch can also affect episodic memory encoding of visually presented items. Following the logic of the ELU framework the higher occurrence of mismatch and concomitant extra processing load in participants with degraded phonological representations in the visual rhyme judgment task is likely to reduce episodic recognition of the rhyme task words. 
WORKING MEMORY COMPENSATES

However, recall performance also differs depending on what strategy was used in the original task. In a recent fMRI-study, Lazard et al (2010) found that participants with acquired hearing impairment relied either on a dorsal, phonological route, or on anterior and ventral networks in a visual rhyme judgment task. Activation of anterior and ventral networks was interpreted as reflecting use of a more global and semantic approach to the written material, in spite of the phonological demands inherent in the task. Use of this semantic strategy predicted poorer speech perception with a cochlear implant. Further, as activity in anterior areas involved in semantic access increased with hearing loss duration in this group, authors suggested that reliance on this semantic route reflected compensation for phonological decline. Attending to semantic rather than phonological features is associated with better episodic recall (Craik, 2002; Craik \& Tulving, 1975; Morris, Branford \& Franks, 1977; Unsworth, Brewer \& Spillers, 2011).

Our second research question, whether hearing related mismatch affect episodic memory encoding, was investigated by testing delayed incidental episodic recognition memory of words presented in the rhyme judgment task.

Our hypothesis was that participants with hearing impairment would have lower recognition scores than normal hearing participants of words from the incongruent rhyme task conditions. If so, that would provide additional support for the ELU model.

\section{Materials and methods}

The data reported below were collected as part of a larger Event-related potentials (ERP) study. ERP-results are under analysis and will be reported separately. 


\section{WORKING MEMORY COMPENSATES}

\subsection{Participants}

Forty-two adults, 6 women, aged 45 to 74 years $(M=65.07, S D=5.60)$ participated in the study. They were all native Swedish speakers and reported normal or corrected-to- normal vision, no psychiatric or neurological disorders and no history of reading disability. Twenty-one individuals ( 3 women) with post-lingually acquired moderate-to-severe sensorineural hearing loss (HI) were recruited from the hearing clinic at Linköping University Hospital. Mean best ear pure tone average measured across 500, 1000, 2000 and $4000 \mathrm{~Hz}$ (BestEarPTA) was 76.67 $(S D=13.87$, range 64-114). Their hearing had been impaired for 37 years on average $(S D=11.01$, range $=10-51)$ and mean age at hearing loss onset was 27 years $(S D=12$, range 6-53). Duration and age of onset were self-reported responses to the question when they first became aware of having a hearing problem. All were bilaterally fitted with hearing aids and the average length of time since fitting was 21 years $(S D=10.93$, range $=4-47)$.

Twenty-one (3 women) normal hearing individuals $(\mathrm{NH})$ were recruited from the general population in the region corresponding to the catchment area of the hearing clinic and constituted the reference group. Normal hearing was here defined as a BestEarPTA of less than $26 \mathrm{~dB}$ hearing loss. Mean BestEarPTA was $15.71(S D=5.86$, range $=3-25)$. NH were matched to HI on age, years in education, non-verbal reasoning ability (Ravens Standard Progressive Matrices, RSPM), verbal ability (word comprehension), WMC (Reading Span), and phonological short-term memory span (digit span forwards). The descriptive data are summarized in Table 1 and there is a full description of the tests used in section 2.3. The study was approved by the regional ethics committee and all participants provided written informed consent before testing started. 
WORKING MEMORY COMPENSATES

Table 1. Means and standard deviations for the normal hearing (NH), hearing impaired (HI), and high/low WMC subgroups on BestEarPTA, age, years in education, RSPM, word comprehension, Reading Span and digit span.

\begin{tabular}{lcccccc}
\hline & \multicolumn{3}{c}{ NH } & \multicolumn{3}{c}{ HI } \\
\cline { 2 - 7 } & NH & NH-High & NH-Low & HI & HI-High & HI-Low \\
& $n=21$ & $n=8$ & $n=9$ & $n=21$ & $n=8$ & $n=9$ \\
& $M(S D)$ & $M(S D)$ & $M(S D)$ & $M(S D)$ & $M(S D)$ & $M(S D)$ \\
\hline Best Ear PTA & $15.71(5.86)$ & $14.63(6.86)$ & $16.89(5.25)$ & $76.67(13.87)$ & $78.88(20.72)$ & $77.56(8.43)$ \\
Age & $66.24(5.65)$ & $65.25(2.55)$ & $68.22(1.99)$ & $63.90(6.24)$ & $59.88(7.68)$ & $67.00(3.00)$ \\
Years in education & $14.69(2.41)$ & $15.25(2.58)$ & $13.72^{\mathrm{a}}(2.33)$ & $13.26(4.66)$ & $15.56(2.81)$ & $9.89^{\mathrm{a}}(2.57)$ \\
RSPM & $50.00(5.66)$ & $52.43(3.51)$ & $47.67(6.30)$ & $46.00(7.78)$ & $50.75(5.75)$ & $41.33(7.62)$ \\
Word comprehension & $31.05(1.56)$ & $31.38(1.06)$ & $30.78(1.99)$ & $30.70(2.18)$ & $31.25(1.67)$ & $30.75(0.89)$ \\
Reading Span & $19.81(4.13)$ & $24.13(2.47)$ & $16.11(1.76)$ & $20.28(5.02)$ & $25.50(3.30)$ & $15.89(2.09)$ \\
Digit span & $59.5(6.5)$ & $60.25(3.92)$ & $57.44(8.33)$ & $56.9(11.5)$ & $64.62(10.80)$ & $52.11(11.32)$ \\
\hline
\end{tabular}

Note: NH-High, normal hearing and high WMC; NH-Low, normal hearing and low WMC; HI-High, hearing impairment and high WMC; HI-Low, hearing impairment and low WMC.

${ }^{\mathrm{a}} \mathrm{p}<.05$ for NH-Low versus HI-Low comparison.

\subsection{General procedure}

Participants were tested at two separate occasions. In the first session a licensed clinical psychologist collected background information and administered a test battery to assess cognitive abilities. The battery included tests of non-verbal and verbal abilities, verbal working memory span, phonological short-term memory, and phonological decoding (see section 2.3.). The rhyme judgment and episodic recognition tasks were presented at the beginning of this session and ERPs were recorded during the rhyme task. Total length of the session was approximately 4 hours, including a 30 minute coffee break and time for applying and removing the ERP net. The rhyme task took around 40 minutes to complete, and the episodic recognition task around 10 minutes. In a second session an experienced audiologist collected audiograms from all participants. 
WORKING MEMORY COMPENSATES

\subsection{Cognitive tests}

All cognitive tests were visually presented. Instructions were oral and care was taken to ensure that all participants correctly understood test procedures.

\subsubsection{Raven Standard Progressive Matrices (RSPM)}

Non-verbal reasoning ability (fluid intelligence) was assessed by RSPM (Raven, Raven \& Court, 2000). RSPM consists of a total of sixty matrices that are divided into five sets. Each matrix shows a pattern from which a piece is missing. Below the matrix there are a number of alternatives and the participant's task is to choose the one which best completes the matrix. There is no time limit. Possible scores range from 0 to a maximum of 60 .

\subsubsection{Word comprehension}

Verbal ability was measured by “Ordförståelse B” (Word comprehension B), a multiple choice, pen and paper, word comprehension test with no time limit. The test is part of the Swedish test battery DLS ${ }^{\mathrm{TM}}$ (Diagnostic tests of reading and writing, Järpsten, 2002). Each of its 34 items is a sentence with the last word missing, for example "Passive is the same as", followed by a list of four words. The task is to choose the word that best completes the sentence. Scores range from 0-34. 
WORKING MEMORY COMPENSATES

\subsubsection{Digit span forward}

Phonological short-term memory was assessed by a computerized digit span forward test. Digits from 1 to 9 were chosen to create random sequences of 2-9 items. Sequence length was progressively increased and there were two trials for each length. Results were scored as number of correct digits in the correct serial position yielding a range of possible scores from 0-88.

\subsubsection{Reading Span}

A Swedish version of the Daneman and Carpenter (1980) Reading Span test was used to assess WMC (Rönnberg, Arlinger, Lyxell \& Kinnefors, 1989). Written sentences were presented word-by-word on a computer screen. After each sentence participants were to report whether the sentence was absurd or not. Half of the sentences were absurd ("The fork often cried") and half were not ("The car went fast"). After having seen a set of sentences, participants were asked to report either the first or the last words of each sentence in the set. Thus, they did not know which words (first or last) they would be asked to report until after they had read the sentences in the set. Number of sentences per set increased progressively from three to six with three trials per set size. The test was scored as the number of words correctly recalled and scores could range from $0-54$.

\subsubsection{Non-word reading}


WORKING MEMORY COMPENSATES

Grapheme-to-phoneme conversion (phonological decoding) was assessed by a test of non-word reading ("Ljuden ger orden", The sounds give the word, Lundberg \& Wolff, 2003). Each item consists of a row of three phonologically legal printed pseudowords, one of which sounds like a real word. The task is to find and underline the ones which sound like real words within a two-minute time limit. Possible scores range from 0-60.

\subsection{Experimental materials}

\subsubsection{Rhyme judgment task}

Stimuli consisted of 192 Swedish word-pairs with 4 different types of congruity. Forty-eight pairs rhymed and were orthographically similar (R+O+, korp - torp, [kår:p] - [tår:p]), 48 rhymed but were orthographically dissimilar (R+O-, helg - välj, [hel:j] - [vel:j]), 48 did not rhyme but were orthographically similar (R-O+, sant - saft, [san:t] - [saf:t]) and 48 neither rhymed, nor were orthographically similar (R-O-, bröd - spik, [brö:d] - [spi:k]). Grapheme-to-phoneme correspondence is more consistent in Swedish than, for example, English (see Seymour et al., 2003) and few orthographically dissimilar Swedish words rhyme. Because of this, word-pairs in the $\mathrm{R}+\mathrm{O}-$ condition included words of low frequency and words from different open word classes (nouns, verbs, adjectives and adverbs). Words for the other conditions were selected to match the $\mathrm{R}+\mathrm{O}-$ words in word frequency, word length, word class, number of syllables and stress pattern to ensure the four conditions did not differ in any of these variables. Specific letter combinations can typically only be pronounced in one way in Swedish. Therefore, Swedish R-O+ word-pairs have orthographically similar, but not identical, final syllables. With the aim of creating similar word-end gestalts a number of strategies were 
WORKING MEMORY COMPENSATES

adopted. In most cases word-endings differed by one letter (e.g. sant - saft, [san:t] - [saf:t]). In other cases letters were rearranged (e.g rost-fots, [rås:t] - [fo:ts]) or added (e.g. besked-beskydd, [befẹ:d] [befyd:]). The variety of solutions reduced the predictability of the orthographic form of the second word in each pair. This ensured the saliency of mismatch in the R-O+ condition.

All words were three to nine letters long, mono- or disyllabic and selected from the Swedish text corpus PAROLE (Språkbanken, University of Gothenburg). The distribution of mono- and disyllabic words, word classes and stress patterns was even over conditions and positions in word-pairs. ANOVAs with Word length and Word frequency as dependent variables, and Condition (4 levels: $\mathrm{R}+\mathrm{O}+, \mathrm{R}+\mathrm{O}-, \mathrm{R}-\mathrm{O}+$ and $\mathrm{R}-\mathrm{O}-$ ) and position ( 2 levels: first word, second word), as independent variables, showed that there were no differences in either word frequency or word length over conditions or positions. All 192 word-pairs were randomized into a single list and this same list was presented to all participants.

\subsubsection{Episodic recognition task}

Two additional lists of words were made for the episodic recognition task. Each contained half of all the words that occurred as the second word in the rhyme judgment wordpairs. To these 96 old words ( 24 from each rhyme condition), an equal number of new words were added, selected from the same text corpus as the rhyme words. The new words were matched to the rhyme words on frequency, word length, word class and proportion of mono- and disyllabic words. The same new words were used in both lists. The two lists were counterbalanced across participants. 
WORKING MEMORY COMPENSATES

\subsection{Procedure}

\subsubsection{Rhyme judgment task}

Words were presented visually one at a time for $200 \mathrm{~ms}$ on a computer screen. Two SOA's were used: $250 \mathrm{~ms}$ (50 ms interstimulus interval) and $1000 \mathrm{~ms}$ (800 ms interstimulus interval). The rhyme judgment list was presented twice to all participants, once with long, and once with short SOA. Order of presentation was counterbalanced. The task was to decide whether the words in each pair rhymed or not and to respond with a button press. Participants were instructed not to pay attention to how words were spelled, but to decide solely on basis of how they are pronounced. A delayed response paradigm was used. A probe appeared on the screen one second after presentation of the second word in the word-pair and signaled that the participant could respond. From probe onset, participants had five seconds to respond. We reasoned that this downplaying of time pressure would ensure that the older participants were not put at a disadvantage as compared to the younger participants. Note that the instruction and the consecutive presentation of the words in each pair (as opposed to simultaneous presentation), encourage a phonological strategy and reduce the effect of conflicting orthographic cues.

\subsubsection{Episodic recognition task}

After the rhyme judgment task there was a short break of approximately 10 minutes before the episodic recognition task was administered. Participants had not been told in advance that their memory of the rhyme words would be tested, just that there would be another task. 
WORKING MEMORY COMPENSATES

Stimuli were presented one at the time on the same computer screen as the rhyme judgment word-pairs. Participants were asked to press the button for "yes" if they thought the word on screen had been presented in the rhyme judgment task and the button for "no" if they did not think so. Again, there was no time limit; words remained on screen until a response was delivered.

\subsection{Statistical analyses}

Repeated measures ANCOVAs with rhyme (2 levels: rhyming, non-rhyming) and orthography (2 levels: orthographically similar, dissimilar) as within participants factors, and hearing status (2 levels: NH, HI) and WMC (2 levels: high, low) as between participants factor, was used to examine results unless otherwise explicitly stated. In the rhyme task, the dependent variable was percent correct judgments. In the incidental episodic recognition task, scores were computed as the proportion correctly recognized old words. For each participant, only words belonging to rhyme task word-pairs that had been correctly judged were included. To examine the effect of SOA, it was included (2 levels, short, long) in the ANCOVA as a within participants factor. Age was entered as a covariate in all analyses (see section 3.1.). Interactions were examined by pairwise t-tests with Bonferroni correction for multiple comparisons. Relationships between variables were examined with partial correlations and predictions were tested with multiple linear regressions, stepwise method.

\section{Results}


WORKING MEMORY COMPENSATES

\subsection{Reading Span and characteristics of the high-and low WMC subgroups}

Mean Reading Span score was $20.05(S D=4.54$, range $=12-31)$. The median, 19.5, was used to divide participants along a high versus low WMC axis. To increase differences in WMC, participants scoring 19 or 20, four NH and four HI, were not included. This resulted in 4 subgroups: normal hearing with high WMC (NH-High, $n=8)$, normal hearing with low WMC (NH-Low, $n=9)$, hearing impaired with high WMC (HI-High, $n=8)$ and hearing impaired with low WMC (HI-Low, $n=9)$.

An ANOVA with hearing status (NH, HI) and WMC (high, low) as independent variables, and Reading Span scores as dependent variable, showed no main effect of hearing status. Nor did hearing status interact with WMC. Thus, there were no differences in WMC between NH-High and HI-High or between NH-Low and HI-Low. Independent samples t-tests showed that HI-High and HI-Low did not differ in any of the hearing measures (BestEarPTA, years since hearing loss onset, or years of bilateral hearing aid usage).

It is inevitable that high and low WMC individuals will also differ on other cognitive measures. As expected those with high WMC were younger $(F(1,30)=11.53, M S E=215.90$, $p=.002)$ and better on $\operatorname{RSPM}(F(1,30)=11.20, M S E=407.68, p=.002)$ and digit span $(F(1.30)=5.10, M S E=83.48, p=.021)$. A Hearing Status $\mathrm{x}$ WMC interaction was only found for the variable years in education $(F(1,30)=5.51, M S E=36.40, p=.026)$. NH-Low had more years in education than HI-Low $(t(30)=4.47, p<.001)$.

In short, the four subgroups remained very similar, except for the lower education level of HI-Low than NH-Low. As the two subgroups did not differ on any of the other variables this was considered acceptable. However, as participants with high WMC were younger, age was 
WORKING MEMORY COMPENSATES

added as a covariate in all analyses conducted on the rhyme judgment and recognition scores reported in sections 3.2 and 3.3 below. There were no significant effects of age in any of the ANCOVAS conducted and therefore age is not further discussed. Descriptive data for the subgroups is summarized in Table 1.

\subsection{Rhyme judgment task}

An initial ANCOVA was conducted to investigate whether SOA had any impact on rhyme judgment performance. There was no main effect of SOA, and no interactions between SOA and hearing status, WMC or rhyme task condition. Thus, data were collapsed over the two SOA's in the analyses below and SOA is not further analyzed.

\subsubsection{Rhyme judgment, percent correct by hearing status and WMC}

A repeated measures ANCOVA was conducted to test the hypothesis that WMC impact rhyme judgment performance in $\mathrm{HI}$ only, and more so in the $\mathrm{R}-\mathrm{O}+$ than the $\mathrm{R}+\mathrm{O}-$ condition. Results showed a main effect of hearing status $(F(1,29)=9.70, M S E=93.18, p=.004)$ as HI made more errors than NH. The significant Hearing Status x Phonology x Orthography interaction $(F(1,29)=6.39, M S E=55.50, p=.017)$ showed that, as expected, this effect was driven by the incongruous conditions. HI made significantly more errors than $\mathrm{NH}$ in both $\mathrm{R}+\mathrm{O}-$ and $\mathrm{R}-\mathrm{O}+\left(t_{\mathrm{R}+\mathrm{O}-}(58)=5.92, p<.001, \mathrm{r}_{\text {contrast }}=.62, t_{R-O+}(58)=2.75, p=.008, \mathrm{r}_{\text {contrast }}=.34\right)$. Hearing status had no impact on performance in the congruous conditions. 
WORKING MEMORY COMPENSATES

The ANCOVA also resulted in a main effect of $\operatorname{WMC}(F(1,29)=9.91, M S E=93.18$, $p=.004)$. Participants with low WMC made more errors than participants with high WMC. This effect was driven by R-O+ which was the only condition in which WMC had an impact (WMC x Phonology x Orthography interaction, $F(1,29)=7.79, M S E=55.50, p=.009, t_{R-O+}(58)=8.80$, $\left.p<.001, \mathrm{r}_{\text {contrast }}=.76\right)$.

The highest order significant interaction was Hearing Status x WMC x Phonology x Orthography $(F(1,29)=4.97, M S E=55.50, p=.034)$. As shown by Fig. 1 , all four subgroups performed close to ceiling level in the congruous conditions ( $\mathrm{R}+\mathrm{O}+$, $\mathrm{R}-\mathrm{O}-)$ while the pattern in the incongruous conditions is more complicated. Post hoc analyses focusing on the incongruous conditions showed that for NH there was no effect of WMC. By contrast, WMC modulated HI performance in a way that differed between the two conditions. In R-O+, HI-High outperformed not only HI-Low $\left(t(58)=10,68, p<.001, \mathrm{r}_{\text {contrast }}=.82\right)$ but also NH-High $(t(58)=2.44, p=.018$, $\left.\mathrm{r}_{\text {contrast }}=.31\right)$. Further, HI-High made significantly more correct judgments in $\mathrm{R}-\mathrm{O}+$ than in $\mathrm{R}+\mathrm{O}-$ $\left(t(29)=8.64, p<.001, \mathrm{r}_{\text {contrast }}=.85\right)$. There was no such difference between the incongruous conditions in NH-High. However, in R+O-, HI-High performed as poorly as HI-Low and made significantly fewer correct judgments than NH-High $\left(t(58)=3.40, p=.001, \mathrm{r}_{\text {contrast }}=.41\right)$. HI-Low made significantly fewer correct judgments than NH-Low in both incongruous conditions $\left(t_{\mathrm{R}+\mathrm{O}-}(58)=5.00, p<.001, \mathrm{r}_{\text {contrast }}=.55, t_{R-O+}(58)=6,59, p<.001, \mathrm{r}_{\text {contrast }}=.66\right)$.

To summarize, WMC modulated rhyme judgment performance in the hearing impaired group but not in the normal hearing group. In $\mathrm{R}+\mathrm{O}-$, the participants with hearing impairment performed worse than the normal hearing participants irrespective of WMC. In R-O+, participants with hearing impairment and good WMC performed better than all the other subgroups, and also significantly better than in $\mathrm{R}+\mathrm{O}-$. By contrast, hearing impaired participants 
WORKING MEMORY COMPENSATES

with low WMC were disadvantaged in both incongruous conditions and outperformed by each of the other subgroups.

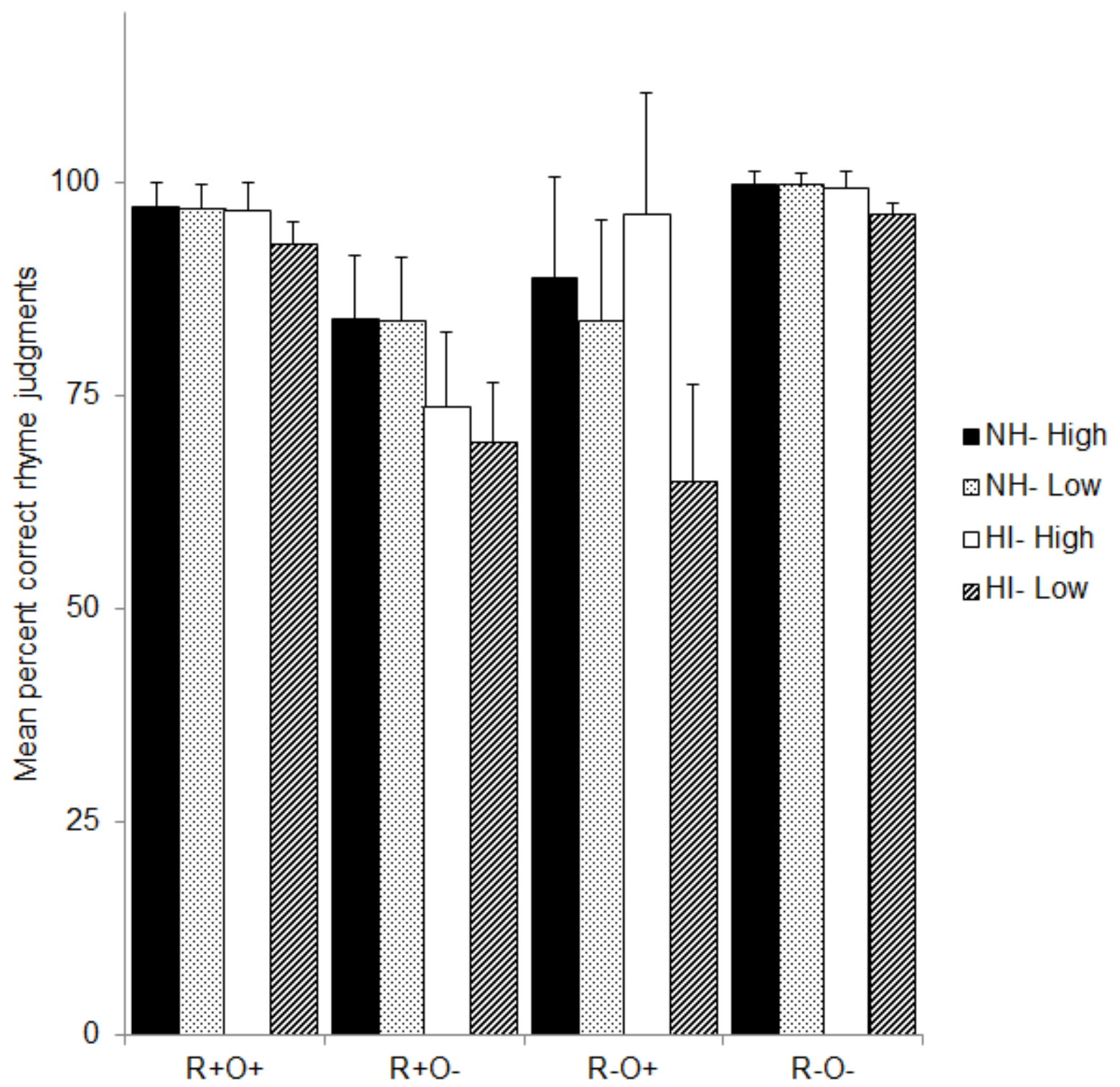

Fig. 1. Mean percent correct rhyme judgments as a function of hearing status, WMC and condition after controlling for age. Error bars represent $95 \%$ confidence interval. 
WORKING MEMORY COMPENSATES

3.2.2. Relationships between duration of bilateral hearing loss, phonological decoding skills and WMC and rhyme judgment performance

As can be seen in Table 2, duration of bilateral hearing loss, measured as number of years using two hearing aids, correlated negatively with rhyme judgment performance in the rhyming conditions. The relationship was strongest in $\mathrm{R}+\mathrm{O}-$, the condition most directly tapping the quality of phonological representations. This result confirms the progressive deterioration of phonological representations in association with acquired hearing loss.

To examine whether phonological decoding skills could explain rhyme judgment performance, an ANCOVA with hearing status and WMC as between participants' factors and non-word reading as dependent variable was conducted. There were significant main effects of hearing status $(F(1,29)=6.32, M S E=66.57, p=.018)$ and $\operatorname{WMC}(F(1,29)=4.83, M S E=66.57$, $p=.036)$. HI scored lower than NH and participants with low WMC scored lower than participants with high WMC. Hearing status did not interact with WMC and thus there were no differences between NH-High and HI-High, or between NH-Low and HI-Low.

Thus, both hearing impairment and low WMC were associated with reduced phonological decoding skills. These results provide further evidence for hearing related phonological processing deficit. However, the lack of Hearing Status x WMC interactions suggests that decoding skill differences could not explain the rhyme judgment results. This conclusion was further corroborated by examination of the correlations between rhyme judgment performance, non-word reading, Reading Span and hearing status (Table 2). As expected, phonological decoding contributed to $\mathrm{R}-\mathrm{O}+$ performance in both the normal hearing and the 
WORKING MEMORY COMPENSATES

hearing impaired participants. However, only in participants with hearing impairment did R-O+ performance also correlate with WMC.

Table 2. Partial correlations coefficients (controlling for age) between duration of bilateral hearing loss, non-word reading, and Reading Span and performance on the rhyme judgment task by hearing status and condition.

$\begin{gathered}\text { Duration of bilateral } \\ \text { hearing loss }\end{gathered} \quad$ Non-word reading $\quad$ Reading Span

\begin{tabular}{cccccc} 
Rhyme judgment & HI & NH & HI & NH & HI \\
\hline R+O+ & $-.62^{\mathrm{b}}$ & -.09 & .23 & .20 & .41 \\
R+O- & $-.75^{\mathrm{a}}$ & -.25 & .08 & .21 & .18 \\
R-O+ & .34 & $.51^{\mathrm{b}}$ & $.53^{\mathrm{b}}$ & -.08 & $.53^{\mathrm{b}}$ \\
R-O- & .23 & .32 & $.65^{\mathrm{a}}$ & -.04 & .41 \\
\hline
\end{tabular}

Note: $\mathrm{NH}$, normal hearing; $\mathrm{HI}$, hearing impairment.

${ }^{\mathrm{a}} p<.01$.

${ }^{b} p<.05$.

In order to explore whether WMC and phonological decoding skills made different contributions to $\mathrm{R}-\mathrm{O}+$ rhyme judgment performance, separate multiple linear regressions were conducted for $\mathrm{HI}$ and $\mathrm{NH}$ with rhyme judgment performance (R-O+) as criterion variable, and age, Reading Span and non-word reading as predictor variables. For NH, only non-word reading predicted $\mathrm{R}-\mathrm{O}+$ performance, explaining $26 \%$ of the variance. For HI, both non-word reading and Reading Span significantly predicted performance and together explained 54\% of the variance (see Table 3).

In short, phonological decoding predicted R-O+ performance in both groups but WMC predicted $\mathrm{R}-\mathrm{O}+$ variance over and above phonological decoding skills only in the hearing impaired group. 


\section{WORKING MEMORY COMPENSATES}

Table 3. Results of linear regression, stepwise method, with R-O+ rhyme judgment performance as criterion variable. $n=21$.

\begin{tabular}{clcccccccc}
\hline & & \multicolumn{3}{c}{$\mathrm{NH}$} & \multicolumn{3}{c}{ HI } \\
Model & & $B$ & $S E B$ & $\beta$ & $R^{2}$ & $B$ & $S E B$ & $\beta$ & $R^{2}$ \\
\hline 1 & Constant & 67.51 & 8.38 & & & 49.18 & 10.41 & & \\
& Non-word reading & 0.73 & 0,29 & $.50^{\mathrm{a}}$ & .26 & 1.35 & 0.40 & $.61^{\mathrm{a}}$ & .37 \\
& & & & & & & & & \\
2 & Constant & - & - & & & 20.26 & 14.68 & & \\
& Non-word reading & - & - & - & & 0.10 & 0.38 & $.45^{\mathrm{b}}$ & \\
& Reading Span & - & - & - & - & 0.10 & 0.40 & $.44^{\mathrm{b}}$ & .54
\end{tabular}

Note: $\mathrm{NH}$, normal hearing; HI, hearing impairment.

${ }^{\mathrm{a}} p<.01$.

${ }^{\mathrm{b}} p<.05$.

\subsection{Episodic recognition: proportion correct by hearing status and WMC}

Repeated measures ANCOVA, testing the hypothesis that higher occurrence of mismatch and extra processing load for $\mathrm{HI}$ in the judgment task lead to lower recognition of words from the incongruous rhyme task conditions, resulted in no main effects. WMC interacted with phonology $(F(1,29)=10.31, M S E=135.50, p=.003)$ as a larger proportion of words from the rhyming than the non-rhyming conditions were recognized $\left(t_{\text {HighWMC }}(29)=8.38, p<.001\right.$, $\left.\mathrm{r}_{\text {contrast }}=.84 ; t_{\text {LowWMC }}(29)=3.38, p=.002, \mathrm{r}_{\text {contrast }}=.54\right)$ and there was a tendency towards lower recognition of words from the rhyming conditions by participants with low WMC $(t(58)=1.89$, $\left.p=.064, \mathrm{r}_{\text {contrast }}=.24\right)$. The Hearing Status $\mathrm{x}$ Phonology $\mathrm{x}$ Orthography interaction was not significant and thus there was no difference between $\mathrm{HI}$ and $\mathrm{NH}$ in recognition rate of words from the incongruous conditions.

However, there was a significant four-way Hearing Status x WMC x Phonology $\mathrm{x}$ Orthography interaction $(F(1,29)=4.89, M S E=112.96, p=.035)$. As can be seen in Fig. 2, 
WORKING MEMORY COMPENSATES

there was a consistent pattern of a higher proportion correctly recognized words in high than low WMC individuals in NH. However, for HI, WMC did not seem to influence performance, but with a notable exception for the R-O+ condition. Here HI-high seems to recognize a lower proportion of words than HI-Low. Post hoc procedures confirmed this: HI-Low outperformed HI-High $\left(t(58)=2.91, p=.005, \mathrm{r}_{\text {contrast }}=.36\right)$ in recognition of $\mathrm{R}-\mathrm{O}+$ words while they performed on a par in $\mathrm{R}+\mathrm{O}-$ - HI-High also showed a trend towards recognizing a lower proportion of $\mathrm{R}-\mathrm{O}+$ words than $\mathrm{NH}-\mathrm{High}$, but this difference did not reach significance after Bonferroni correction $\left(t(58)=2.06, p=.044, \mathrm{r}_{\text {contrast }}=.26\right)$. 
WORKING MEMORY COMPENSATES

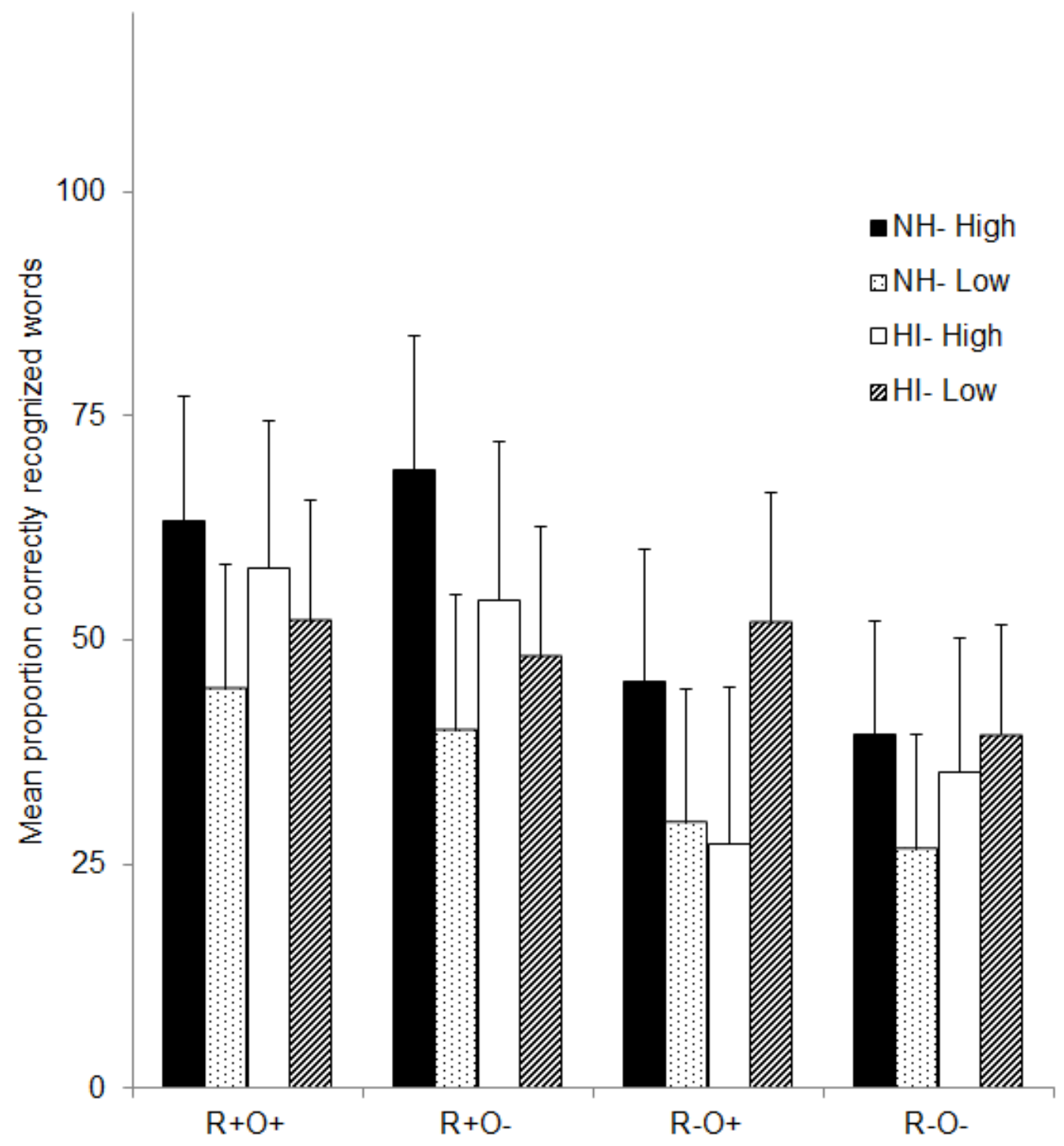

Fig. 2. Mean proportion correctly recognized words from correctly judged rhyme task word-pairs as a function of hearing status, WMC and condition after controlling for age. Error bars represent $95 \%$ confidence interval.

To summarize, the hypothesis that hearing status would impact recognition of words from the incongruous conditions was not supported. Instead, there was the surprising result that in the hearing impaired participants, WMC modulated R-O+ performance such that low WMC was associated with a higher recognition rate than high WMC. 
WORKING MEMORY COMPENSATES

\section{Discussion}

The results of the present study provide additional evidence that acquired hearing loss is associated with progressive deterioration of phonological representations. Hearing impaired participants performed worse than normal hearing participants in non-word reading and rhyme judgments of word-pairs with conflicting orthographic cues. Rhyme detection accuracy decreased with duration of hearing loss severe enough to prompt the use of bilateral hearing aids. This is in line with the previous literature (Andersson, 2002; Andersson \& Lyxell, 1998; Lazard et al., 2010; Lee et al., 2007; Lyxell et al., 1998; Lyxell et al., 2003; Lyxell et al., 1993). The novel findings in this study are the hearing related interactions between WMC and phonological processing ability and between WMC and episodic recognition memory. These interactions are discussed below.

\subsection{Rhyme judgment task}

In the normal hearing participants, rhyme judgment performance was independent of WMC. This indicates that with access to precise phonological representation, working memory load need not be very high in this task. Indeed, although working memory is often implicated in phonological awareness tasks that, like rhyme judgment, require extraction, storage and manipulation of phonemes or syllables (Oakhill \& Kyle, 2000), working memory and phonological processing load on different factors in factor analyses (Alloway, Gathercole, Willis \& Adams, 2004) and make separate contributions to reading development (for a review, see Savage, Lavers \& Pillay, 2007). 
WORKING MEMORY COMPENSATES

By contrast, in the hearing impaired group, high WMC was associated with significantly better performance in the orthographically similar non-rhyming condition. As described in the introduction, the two incongruous rhyme task conditions differ from each other. Judgments of orthographically dissimilar rhymes more directly tap the precision of phonological representations (Andersson, 2002) while judgments of orthographically similar non-rhymes to a greater extent also involve performing phonological operations and analyses (Andersson, 2002; Johnston \& McDermott, 1986; Rugg \& Barrett, 1987). When the task primarily required access to well defined phonological representations, individuals with hearing impairment performed worse than the normal hearing participants, irrespective of WMC. This shows that access to good working memory does not counteract hearing-related phonological processing decline. However, when efficient phonological processing skills could to a larger extent be used to support performance, i.e. in the orthographically similar non-rhyming condition, the hearing impaired participants with high WMC outperformed the other subgroups, including the normal hearing participants with high WMC. In this condition, word phonology could to a larger degree be accessed by phonological decoding skills and indeed, there was a positive correlation between non-word reading and rhyme judgment performance in this condition. However, in the participants with hearing impairment, WMC explained variance over and above phonological decoding skills. Thus, other processes supported performance.

In rhyme judgment, the relevant information is in the phonological similarity between word-pairs. In this study, a phonological strategy was further encouraged by the task itself, the explicit instructions and the consecutive presentation of words (orthographic interference is larger when both words in a pair are visible on screen, see Johnstone \& McDermott, 1986). With presentation of the first word in a pair, a phonological strategy will lead to generation of an 


\section{WORKING MEMORY COMPENSATES}

expectancy set involving lexical candidates that rhyme with the first word. This will facilitate judgment of rhymes because the expectancy set will include candidates phonologically matching the second word in the pair (Hillinger, 1980; Johnstone \& McDermott, 1986). The independence of WMC in the orthographically dissimilar rhyming condition is suggested to occur because as participants focus on word phonology, they will be better able to confirm the rhyme without a retake or extra comparison. However, this is exactly what is needed in the orthographically similar non-rhyming condition: the words are rather similar in sound, and spelling, but don't rhyme. It is likely that the hearing-impaired participants with high WMC were better than the hearing impaired participants with low WMC at compensating for their relatively poor phonological representations of a rather sound-similar comparison by repeated double-checking of spelling and sound. For example, when detecting the phonological mismatch, they might have chosen an orthographic strategy, comparing word-final spelling. Words were presented on screen one at a time and only for $200 \mathrm{~ms}$. Therefore any such double-checking procedure had to be carried out on stored phonological and orthographic representations, requiring access to efficient explicit resources. Indeed, hearing impaired participants with good WMC were even better than normal hearing participants with good WMC in this condition. This suggests that experience with solving phonological mismatch can improve efficiency of WMC deployment in such conditions. The hearing impaired participants with low WMC were instead outperformed by the other subgroups, including normal hearing participants with low WMC.

Whether there was a short $(50 \mathrm{~ms})$ or a long $(250 \mathrm{~ms})$ time interval between onsets of the two words in the word-pairs had no impact on rhyme judgment performance and did not interact with either hearing status or WMC. WMC could have been expected to be more decisive to performance when the time interval between the words was large enough to allow for generation 
WORKING MEMORY COMPENSATES

of expectancy sets; however this was not the case. A possible explanation lies in the delayed response paradigm used. Participants had time to engage explicit processes after presentation of the word-pair but before responding. A more direct measure of brain activation or a speeded response paradigm would likely be more sensitive to the SOA manipulation.

To summarize, results on the rhyme task support the ELU model: WMC modulated performance in the hearing impaired group only, i.e. where the occurrence of mismatch is proposed to be more frequent, and could be employed to overcome the effect of mismatch.

\subsection{Incidental episodic recognition}

In the normal hearing participants, high WMC was associated with better episodic recognition of correctly judged words across the four rhyme task conditions. This is in line with previous research: better WMC is typically linked to more efficient retrieval and encoding processes and high WMC individuals usually outperform low WMC individuals in recall tasks irrespective of whether encoding is intentional (Unsworth et al, 2011) or incidental (Unsworth \& Spillers, 2010).

There were no differences between hearing and hearing impaired participants in the incongruous conditions. Thus, contrary to our hypothesis, there was no straightforward relationship between hearing-related mismatch (as indicated by lower rhyme task performance) and recognition performance. Instead, there was an interesting interaction between WMC and condition in the hearing impaired group: individuals with high WMC recognized fewer correctly judged words from the orthographically similar non-rhyming condition than individuals with low WMC. Thus, in this condition and group, participants with high WMC performed exceptionally 
WORKING MEMORY COMPENSATES

well in the judgment task, but later recognized few of the words while participants with low WMC performed poorly in the judgment task but later recognized a surprisingly large proportion of the words. It is suggested that, with good WMC, repeated double checking of spelling and sound in working memory can help compensate for mismatch but will also occupy explicit resources and leave fewer available for storage processes. Additionally, if high WMC individuals relied more on orthographic cues, such "shallower" processing would contribute to reduced memory encoding and subsequent recognition.

The result pattern of the hearing impaired participants with low WMC indicates they used a different strategy. As discussed in the introduction, it has been found that individuals with acquired hearing impairment rely either on a phonological route, reflected by activation of dorsal brain networks, or a more global semantic route, activating anterior and ventral networks, in visual rhyme judgments (Lazard et al, 2010; Lazard, Giraud, Gnansia, Meyer, \& Sterkers, 2012). The phonological route, involving application of grapheme-to-phoneme correspondences, is likely to be more working memory demanding. Resorting to a whole-word, semantically based route to word phonology might well be an adaptive strategy to compensate for the progressive deterioration of speech sound representations (Lazard et al, 2010, Lazard et al, 2012), especially for individuals with low WMC, but is likely to interfere with rhyme judgments. However, as attending to semantic, rather than phonological, features of words is also associated with a more durable memory encoding (Craik \& Tulving, 1975; Morris, Branford \& Franks, 1977; Unsworth et al., 2011) such a strategy could enhance recall. Use of a sematic approach in the rhyme task in this study could thus explain both low rhyme judgment performance and high recognition performance and it is suggested that the hearing impaired participants with low WMC, in 
WORKING MEMORY COMPENSATES

adapting to the combination of low quality phonological representations and limited access to explicit resources, resorted to this less cognitively demanding semantic reading strategy.

\subsection{Clinical implications}

Taken together, the present results indicate that the role of cognitive factors in compensating for auditory deprivation is more far-reaching than previously thought. It is known that WMC affects speech understanding under challenging circumstances as well as the capacity to benefit from modern hearing aids (Gatehouse, Naylor \& Elberling, 2006; Lunner \& Sundewall-Thorén, 2007; Rudner et al, 2009). The present results show that in individuals with hearing impairment, cognition also impact non-auditory phonological processing and factors related to reading. Importantly, the double disadvantage encountered by individuals with sensory impairment and lower WMC needs to be fully appreciated in rehabilitation settings. Routine assessment of cognitive abilities in audiology clinics would help ensure that the needs of these individuals are met.

\section{Conclusion}

In support of the ELU model, the present results extend findings from previous studies: explicit resources play an important role in overcoming hearing related mismatch not only in situations where the linguistic input is compromised, but also in situations where the linguistic input is optimal but phonological representations degraded. Taken together, the current results suggest that WMC-related differences in phonological processing abilities in individuals with 
WORKING MEMORY COMPENSATES

severe acquired hearing impairment lead to use of different strategies in phonologically

challenging tasks. In turn, these strategies have different effects on episodic memory encoding. 
WORKING MEMORY COMPENSATES

\section{References}

Alloway, T. P., Gathercole, S. E., Willis, C., \& Adams, A-M. (2004). A structural analysis of working memory and related cognitive skills in young children. Journal of Experimental Child Psychology, 87, 85-106.

Andersson, U. (2002). Deterioration of the phonological processing skills in adults with an acquired severe hearing loss. European Journal of Cognitive Psychology, 14, 335-352.

Andersson, U., \& Lyxell, B. (1998). Phonological deterioration in adults with an acquired severe hearing impairment. Scandinavian Audiology, 27(Suppl 49), 93-100.

Andersson, U., Lyxell, B., Rönnberg, J., \& Spens, K.-E. (2001). Cognitive correlates of visual speech understanding in hearing-impaired individuals. Journal of Deaf Studies and Deaf Education, 6, 103-115.

Baddeley, A. D. (2000). The episodic buffer: a new component of working memory? Trends in Cognitive Sciences, 4(11), 417-423.

Baltes, P. B., \& Lindenberger, U. (1997). Emergence of a powerful connection between sensory and cognitive functions across the adult life span: A new window to the study of cognitive aging? Psychology and Aging, 12, 12-21.

Baum, S. R., \& Leonard, C. L. (1999). Automatic versus strategic effects of phonology and orthography on auditory lexical access in brain-damaged patients as a function of interstimulus interval. Cortex, 35, 647-660.

Craik, F. I. M. (2002). Levels of processing: Past, present... and future? Memory, 10, 305-318.

Craik, F. I. M., \& Tulving, E. (1975). Depth of processing and the retention of words in episodic memory. Journal of Experimental Psychology: General, 10 (3), 268294. 
WORKING MEMORY COMPENSATES

Daneman, M., \& Carpenter, P. A. (1980). Individual differences in working memory and reading. Journal of Verbal Learning and Verbal Behavior, 19, 450-466.

Daneman, M., \& Merikle, P. M. (1996). Working memory and language comprehension: A meta-analysis. Psychonomic Bulletin \& Review, 3(4), 422-433.

Gatehouse, S., Naylor, G., \& Elberling, C. (2006). Linear and nonlinear hearing aid fittings - 2 . Patterns of candidature. International Journal of Audiology, 45, 153-171.

Hillinger, M. L. (1980). Priming effects with phonemically similar words: The encoding bias hypothesis reconsidered. Memory \& Cognition, 8, 115-123.

Johnston, R. S., \& McDermott, E. A. (1986). Suppression effects in rhyme-judgement tasks. Quarterly Journal of Experimental Psychology, 38A, 111-124.

Järpsten, B. (2002). DLS ${ }^{T M}$ handledning. Stockholm: Hogrefe Psykologiförlaget AB

Kramer, A. F., \& Donchin, E. (1987). Brain potentials as indices of orthographic and phonological interactions during word matching. Journal of Experimental Psychology: Learning, memory and Cognition, 13, 76-86.

Lazard, D. S., Giraud, A. L, Gnansia, D., Meyer, B, Sterkers, O. (2012). Understanding the deafened brain: Implications for cochlear implant rehabilitation. European Annals of Otorhinolaryngology, Head and Neck diseases, 129, 98-103.

Lazard, D. S., Giraud, A. L., Truy E., \& Lee, H. J. (2011). Evolution of non-speech sound memory in postlingual deafness: Implications for cochlear implant rehabilitation. Neuropsychologia, 49, 2475-2482.

Lazard, D. S., Lee, H. J., Gaebler, M., Kell, C. A., Truy, E., \& Giraud, A. L. (2010). Phonological processing in post-lingual deafness and cochlear implant outcome. NeuroImage, 49, 3443-3451. 
WORKING MEMORY COMPENSATES

Lee, H. J., Truy, E., Mamou, G., Sappey-Marinier, D., \& Giraud, A. L. (2007). Visual speech circuits in profound acquired deafness: s possible role for latent multimodal connectivity. Brain, 130, 2929-2941.

Luce, P. A., \& Pisoni, D. A. (1998). Recognizing spoken words: the neighborhood activation model. Ear \& Hearing, 19, 1-36.

Lundberg, I., \& Wolff, U. (2003). DUVAN ${ }^{T M}$. Dyslexiscreening för ungdomar och vuxna. Stockholm: Psykologiförlaget AB.

Lunner, T. (2003). Cognitive function in relation to hearing aid use. International Journal of Audiology, 42(Suppl. 1), 49-58.

Lunner, T., \& Sundewall-Thorén, E. (2007). Interactions between cognition, compression, and listening conditions: Effects on speech-in-noise performance in a two-channel hearing aid. Journal of the American Academy of Audiology, 18, 604-617.

Lyxell, B., Andersson, J., Andersson, U., Arlinger, S., Bredberg, G., \& Harder, H. (1998). Phonological representation and speech understanding with cochlear implants in deafened adults. Scandinavian Journal of Psychology, 39, 175-179.

Lyxell, B., Andersson, U., Borg, E., \& Ohlsson, I-S. (2003). Working memory capacity and phonological processing in deafened adults and individuals with a severe hearing impairment. International Journal of Audiology, 42, S86-S89.

Lyxell, B., Rönnberg, J., \& Samuelsson, S. (1993). Internal speech functioning and speechreading in deafened and normal hearing adults. Scandinavian Audiology, 23, 179-185.

Marslen-Wilson, W. (1987). Functional parallelism in spoken word recognition. Cognition, 25, 71-103. 
WORKING MEMORY COMPENSATES

McClelland, J. L., \& Elman, J. L. (1986). The TRACE model of speech perception. Cognitive Psychology, 18, 1-86.

McCoy, S. L., Tun, P. A., Cox, C. L., Colangelo, M., Stewart, R. A., \& Wingfield, A. (2005). Hearing loss and perceptual effort: Downstream effects on older adults' memory for speech. The Quarterly Journal of Experimental Psychology, $58 A$ (1), 22-33.

McQueen, J. M., \& Serrano, J. (2005). Cleaving automatic processes from strategis bias in phonological priming. Memory and Cognition, 33, 1185-1209.

Morris, C. D., Bransford, J. D., \& Franks, J. J. (1977). Levels of processing versus transfer appropriate processing. Journal of verbal learning and verbal behavior, 16, 519-533.

Neely, J. H. (1977). Semantic priming and retrieval from lexical memory: Roles of inhibitionless spreading activation and limited-capacity attention. Journal of Experimental Psychology, $106,226-254$.

Neely, J. H., Keefe, D. E., \& Ross, K. L. (1989). Semantic priming in the lexical decision task: Roles of prospective prime-generated expectancies and retrospective semantic matching. Journal of Experimental Psychology: Learning, Memory, and Cognition, 15, 1003-1019.

Ng, E. H. N., Rudner, M., Lunner, T., Syskind Pedersen, M., \& Rönnberg, J. (submitted). Effects of noise and individual differences in working memory capacity on memory processingof speech for hearing aid users.

Oakhill, J., \& Kyle, F. (2000). The relation between phonological awareness and working memory. Journal of Experimental Child Psychology, 75, 152-164.

Pearman, A., Friedman, L., Brooks, J. O., \& Yesavage, J. A. (2000). Hearing impairment and serial word recall in older adults. Experimental Aging Research, 26, 383-391. 
WORKING MEMORY COMPENSATES

Piquado, T., Cousins, K. A. Q., Wingfield, A., \& Miller, P. (2010). Effects of degraded sensory input on memory for speech: Behavioral data and a test of biologically constrained computational models. Brain Research, 1365, 48-65.

Polich, J., McCarthy, G., Wang, W. S., \& Donchin, E. (1983). When words collide: Orthographic and phonological interference during word processing. Biological Psychology, 16, 155-180.

Raven, J., Raven, J. C., \& Court, J. H. (2000). Manual for Raven's Progressive Matrices and vocabulary scales. Section 3, The standard progressive matrices. Oxford, England: Oxford Psychologists Press.

Rudner, M., Foo, C., Rönnberg, J., \& Lunner, T. (2007). Phonological mismatch makes aided speech recognition in noise cognitively taxing. Ear \& Hearing, 28(6), 879-892.

Rudner, M. \& Rönnberg, J. (2008). The role of the episodic buffer in working memory for language processing. Cognitive Processing, 9, 19-28.

Rudner, M., Rönnberg. J. \& Lunner, T. (2011). Working memory supports listening in noise for persons with hearing impairment. Journal of the American Academy of Audiology, 22, $156-167$.

Rugg, M. D., \& Barrett, S. E. (1987). Event-related potentials and the interaction between orthographic and phonological information in a rhyme judgment task. Brain and Language, 32, 336-361.

Rönnberg, J. (2003). Cognition in the hearing impaired and deaf as a bridge between signal and dialogue: A framework and a model. International Journal of Audiology, 42, S68-S76. 
WORKING MEMORY COMPENSATES

Rönnberg, J., Arlinger, S., Lyxell, B., \& Kinnefors, C. (1989). Visual evoked potentials: Relation to adult speechreading and cognitive function. Journal of Speech and Hearing Research, $32,725-735$.

Rönnberg, J., Danielsson, H., Rudner, M., Arlinger, S., Sternäng, O., Wahlin, Å. \& Nilsson, L-G. (2011). Hearing loss is negatively related to episodic and semantic long-term memory but not to short-term memory. Journal of Speech, Language, and Hearing Research, 54, 705-726.

Rönnberg, J., Rudner, M., Foo, C., \& Lunner, T. (2008). Cognition counts: A working memory system for ease of language understanding (ELU). International Journal of Audiology, 47, S171-S177.

Rönnberg, N., Rudner, M., Lunner, T. \& Stenfelt, S. (submitted). Assessing listening effort by measuring short-term storage and processing of speech in noise.

Savage, R., Lavers, N., \& Pillay, V. (2007). Working memory and reading difficulties: What we know and what we don't know about the relationship. Educational Psychology Review, $19,185-221$.

Seymour, P. H. K., Aro, M., Erskine, M. (2003). Foundation literacy acquisition in European orthographies. British Journal of Psychology, 94, 143-174.

Stenfelt, S., \& Rönnberg, J. (2009). The signal-cognition interface: Interactions between degraded auditory signals and cognitive processes. Scandinavian Journal of Psychology, 50(5), 385-393.

Surprenant, A. M. (2007). Effects of noise on identification and serial recall of nonsense syllables in older and younger adults. Aging, Neuropsychology, and Cognition, 14, 126-143. 
WORKING MEMORY COMPENSATES

Språkbanken vid Göteborgs universitet. Web site: http://spraakbanken.gu.se.

Tulving, E. (2002). Episodic memory: From mind to brain. Annual Review of Psychology, 53, 125.

Tun, P. A., McCoy, S., \& Wingfield, A. (2009). Aging, hearing acuity, and the attentional costs of effortful listening. Psychology and Aging, 3, 761-766.

Unsworth, N., Brewer, G. A., \& Spillers, G. J. (2011). Variation in working memory capacity and episodic memory: Examining the importance of encoding specificity. Psychonomic Bulletin \& Review, 18, 1113-1118.

Unsworth, N., \& Spillers, G. J. (2010). Variation in working memory capacity and episodic recall: The contributions of strategic encoding and contextual retrieval. Psychonomic Bulletin \& Review, 17, 200-205.

Valentijn, S. A. M., van Boxtel, M. P. J., van Hooren, S. A. H., Bosma, H., Beckers, H. J. M., Ponds, R. W. H. M., \& Jolles, J. (2005). Change in sensory functioning predicts change in cognitive functioning: Results from a 6-year follow-up in the Maastricht aging study. Journal of American Geriatrics Society, 53, 374-380. 